\title{
Study Closed to Accrual and Treatment
}

National Cancer Institute

\section{Source}

National Cancer Institute. Study Closed to Accrual and Treatment. NCI Thesaurus. Code C70752.

A clinical study status designating the study that stopped further patient accrual as well as the treatment of the subjects already enrolled in the trial. 